Article

\title{
Learning from Incidents: A Supply Chain Management Perspective in Military Environments
}

\author{
Raffaele Cantelmi ${ }^{1,2}$, Giulio Di Gravio ${ }^{1}$ (D) and Riccardo Patriarca ${ }^{1, *(\mathbb{C})}$ \\ 1 Department of Mechanical and Aerospace Engineering, Sapienza University of Rome, Via Eudossiana, \\ 18-00184 Rome, Italy; raffaele.cantelmi@uniroma1.it (R.C.); giulio.digravio@uniroma1.it (G.D.G.) \\ 2 Land Armaments Directorate, Ministry of Defence, Via di Centocelle, 301-00187 Rome, Italy \\ * Correspondence: riccardo.patriarca@uniroma1.it; Tel.: +39-0644585252
}

Received: 27 June 2020; Accepted: 15 July 2020; Published: 17 July 2020

\begin{abstract}
Supply chain management (SCM) represents a crucial role in the military sector to ensure operation sustainability. Starting from the NATO handbook for military organizational learning, this paper aims at investigating the link between technical inconveniences and sustainable supply chain operations. Taking advantage of the learning from incidents (LFI) models traditionally used in the risk and safety management area, this paper proposes an information management system to support organizational learning from technical inconveniences in a military supply chain. The approach is discussed with reference to the Italian context, in line with international and national standards for technical inconvenience reporting. The results of the paper show the benefits of adopting a systematic LFI system for technical inconveniences, providing related exemplar business intelligence dashboards. Further implications for the generalization of the proposed information management system are presented to foster a healthy and effective reporting environment in military scenarios.
\end{abstract}

Keywords: incidents learning system; technical inconveniences; military supply chain; information management; business intelligence

\section{Introduction}

Supplying goods or services to the customer is the main target for a sustainable supply chain network that includes manufacturers, distributors, logistics firms, and many other indirect agents (banks, brokers, insurance companies, etc.) [1]. Until the 1950s, in military environments, similarly to any other production domain, business leaders thought of logistics as a combination of procurement, maintenance, transportation of facilities, material, and personnel [2]. In the early 1980s, such perspective extended to the concept of supply chain management (SCM), widening the idea of logistics to the management of suppliers and customers interactions. SCM refers to a combination of activities undertaken within the organizations to encourage the efficient management of their supply chain, and this caught a lot of attention recently both by academics as well as practitioners [3,4]. SCM has become one of the most popular concepts in the management area since the 1980s [5], with a number of journals in manufacturing, procurement, and transportation, information pushing forward the development of the SCM idea in a global competitive environment [6-8]. Specifically in the military context, the annual industrial capabilities report, put out by the Pentagon's Office of Manufacturing and Industrial Base Policy, acknowledges that the industrial base of the weaponry sector is particularly strained, due to the irregular flow of procurement and the lack of new designs being internally developed [9]. Such observations confirm the critical foundation of measuring and managing information on key operational and performance parameters [10].

In a systemic perspective, it is important to highlight how the delivery of a good or a service does not terminate the supply chain. A systemic SCM shall rather include the so-called reverse 
logistics, which ensure that used, defective and discarded products are managed in order to increase the effectiveness of the network [1]. In the domain of repairable items, reverse logistics is usually regulated by performance-based contracting, i.e., after-sales services aimed at cutting fixed-price costs by ad-hoc interventions [11], following its introduction in the early 1990s [12]. In military contexts, reverse logistics ensure that the material lifecycle is sustainable, facilitating the recycling or reusing of equipment which can be repaired and utilized [13].

Therefore, with the aim of improving SCM sustainability, it has become necessary to adopt structured and effective frameworks for managing reverse streams of the supply chain, both in terms of operations and information management [14]. Good decisions are based on timely, accurate and relevant information, which represent the link between activities and operations [15]. Despite recent significant advances and dramatic improvements in information technology, the discipline of SCM can be further enhanced to address modern practical real-world challenges [16], even more specifically in military supply chains. Available research mostly focuses on the quantitative analysis of detailed aspects of the supply chain. For example, some scholars propose a game model of military reverse logistics based on the Nash equilibrium [17]. Similarly, another work focuses on an optimal model of principal-agent relationships for waste military air materials, stressing the need for provider-specific incentive mechanisms [18]. More recently, a hybrid machine learning model has been developed to improve and predict spare parts reverse flow [19]. A large set of research is also focused on routing problems for reverse logistics or end of life vehicles, exploring multiple facility location problems, e.g., single-facility [20], grouped location problem [21], k-location routing [22], multi-period location routing problems [23]. While these contributions contribute widely on the progress of reverse logistics, they do not present a deep analysis on the management of the reverse flow, especially within military supply chains.

On this side, this paper complements the wide literature relying on mathematical formulation for reverse logistics via a framework to be used for dealing with technical incidents from an organizational perspective. The outcome of the framework allows for an understanding of the strengths and weakness of both the product at hand and the agents involved in its management process.

Based on these premises, it could be possible to relate information on reverse logistics with the theory of learning from incident (LFI), to take maximum advantage from reporting actions.

LFI (or more generally from events) can be defined as a change in the repertoire of behaviors of an organization [24], i.e., a shared understanding of the need for new actions to minimize or prevent negative events [25]. The academic field of LFI is partly fragmented [26], but it is widely acknowledged its potential for both safety and productivity $[27,28]$. For gathering its benefits, learning has to be set in an organizational environment including the identification of events to be investigated, the application of most suitable analysis techniques, a thorough reflection on the results for developing meaningful repertoires, the sharing of these latter, and the structure of the most advantageous conditions for their applicability [25].

LFI is thus aligned with the idea of a sustainable supply chain, where the application of a systematic learning system may provide savings on input costs such as time, labor and energy, and thus enable profitable outputs and increase customer satisfaction, also in light of environmental dimensions [29]. The relevance of such a proactive learning system has been widely acknowledged in military technical publications, as detailed in the NATO lessons learned handbook [30].

Following the theory of LFI [31,32], this paper aims to present the structure of a database to be used in a military environment for the management of technical inconveniences. By exemplary statistics and dashboards to support decision-making, the paper aims to prove the benefits of a systematic LFI information management system. In summary, the purpose of the paper is two-fold: (i) the definition of concepts and roles for implementing an LFI in military environments; (ii) the development of a database structure for ensuring the implementation of previously mentioned roles and concepts, in line with NATO guidelines. 
The remainder of the paper is organized as follows. Section 2 describes the theoretical foundation of an LFI system and describes the relevant military publication both in the international context (i.e., NATO) and for the supply chain of the item at hand (small arms in Italy). Section 3 details the information management system developed in this research and provides exemplar results of the approach. Note that the quantitative results in this section do not refer to the operational situation at hand, but they rather include exemplary anonymized data. Section 4 discusses the obtained results and the advantages of the approach. Lastly, the conclusions summarize the outcome of the approach and provide evidence for further research.

\section{Materials and Methods}

This section describes the theoretical foundation for the LFI system to be used for information management (Section 2.1), subsequently describing (Section 2.2) the regulatory reporting framework of interest for the approach presented in this document (ILE-NL-1110-0001-12-00B01, ILE-NL-21000006-12-00B01), as developed by the entity for logistics (called ILE). Lastly, the section details the as-is reporting framework (Section 2.3).

\subsection{The Theoretical Model}

In our work, we considered a theoretical model inspired by literature regarding incident learning originating from reliability and safety management [31,32]. In such a model, the components of an Incident Learning System include 8 main phases generally (Figure 1): identification and response; reporting; investigation; identifying the causal structure; making recommendations; communicating; recalling incident learnings; implementing corrective actions.

The first component of the LFI system is the "identification and response", without which no learning, even no business survival, is possible [33]. Such observation is not trivial, since unless the organization is already sensitized about a LFI management policy, deviations from normal behavior may not be noticed or will usually be accepted as "normal deviations" (Dekker, 2011), so it is very important to establish an appropriate identification threshold.

The second component of LFI system is "reporting": no incident can be investigated if it is not duly reported. Here, a proper dimension of reporting has to be arranged, containing relevant information, and excluding those which would require too much efforts, jeopardizing even the credibility of the entire report [34].

The "investigation" is a core component of the LFI system. Starting from the outcomes of "reporting" this phase includes the examination of the report, as well as the involved item/site (if needed), and the interview of users/witnesses. This phase aims to collect and evaluate the available data to establish the sequence of events and establish the basis for subsequent causal analyses. It is often claimed that the purpose of incident investigation is to determine the root causes of the incident [35]. However, since there may be no single root cause, efforts would be better directed towards "identifying the causal structure". For incidents that occur in complex systems, one possible approach is to integrate the techniques of analysis of the root causes with system thinking [36,37]. The work of the investigation team is usually considered as completed with the issuance of an incident report detailing the findings and recommendations ("making recommendations"). The dissemination of this document as well as other communication activities within the organization, i.e., "communication", is a means to enhance organizational learning.

A further inherent phase of the learning process is the "recalling incident learnings", i.e., the application of the learning coming from incidents, and the "failure hypothesizing", i.e., using the investigation report to explore possible failure modes that have not yet occurred [38-40].

Lastly, it is important to implement corrective actions and all the recommendations made by the investigative team in order to obtain a cyclic effective LFI system. 


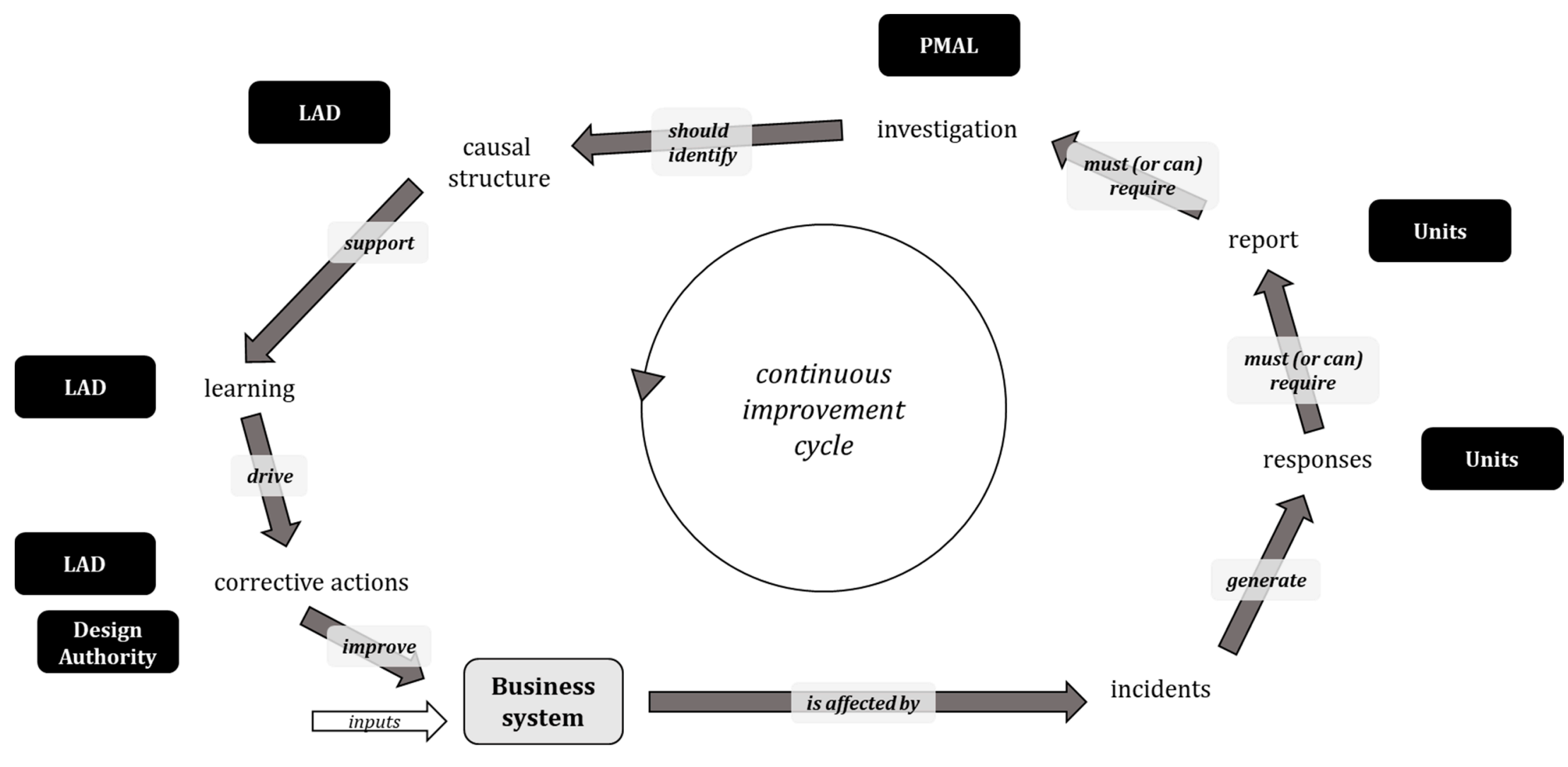

Figure 1. The theoretical learning from incidents (LFI) model. 


\subsection{The Regulatory Framework}

NATO stresses the importance of a LFI model, as established by the Joint Analysis and Lessons Learned Center (JALLC), whose mission is to carry out an inter-force analysis of everyday operations, training, exercises and experiments, including the implementation and management of a single database for all NATO countries regarding lessons learned. The NATO learning doctrine applies to any military activity, even though in real practices it faces the challenges imposed by the lack of a healthy reporting culture. For this purpose, individual armed forces adjust the NATO doctrine on LFI by a set of customized inter-organizations and intra-organization directives, issued by national bodies. On this basis, this paper focused on the regulatory framework implemented in Italy for small arms, as described by two logistics publications which are of interest for sustainable SCM:

- ILE-NL-1110-0001-12-00B01 (see Section 2.2.1), which deals with the management of the different materials supplied to the Italian Army, for the sake of clarity called ILE-MM (ILEMaterial Management)

- ILE-NL-2100-0006-12-00B01 (see Section 2.2.2), which derives from the previous one and regulates the management activities and the logistic device related to the armament material, for the sake of clarity called ILE-LM (ILE-Logistics Management).

The regulatory framework is completed by the technical directive TER-50-1000-0007-12-00B000 (see Section 2.2.3), repeatedly recalled by the ILE-NL-2100-0006-12-00B01, which defines the control procedures to ensure the usability of a weapon in safe conditions for the operator and the reliability for operational purposes.

\subsubsection{ILE-NL-1110-0001-12-00B01}

The publication was issued to maintain the availability of means, materials and weapon systems at the levels set by the Army General Staff, for the effective use of units in operations and training activities. The ILE-MM defines the correct procedures of technical-logistical management so that maintenance interventions and supply activities take place in a harmonious and balanced functional framework, following a cost-effective target. The purpose of the publication is therefore to establish the rules governing the management of the material parks supplied to the Italian Army, for operational, training and logistical needs.

The publication consists of a "general section" and the "park material section". The former provides the main features of the logistic support in all its aspects, and a series of dossiers related to the detailed management rules for homogeneous sets of materials. This section further defines the procedures relating to the main elements of the logistic support, i.e., procurement, supplies, maintenance, transport.

Procurement is at the origin of the material management process and it aims at acquiring the means and materials necessary to achieve or maintain the expected operational capacity.

The Supplies are intended to make the material resources available to the armies, in scheduled times, places and quantities to ensure the operational capabilities necessary for operations. This activity also includes the reverse material handling about replacement of inefficient vehicles and materials.

Maintenance is the activity which seeks to ensure the reliability of the means and materials through checks, revisions, reconfigurations and various types of processing. Furthermore, the ILE-MM specifies the tasks of the Second Level of Maintenance (FLS) institutions, i.e., the Maintenance Poles, which are entrusted with all the corrective actions that fall outside the competence of the First Level of Maintenance (FLA) and the general revision interventions related to the peculiarity of the materials, means and weapon systems. Moreover, the publication establishes, among a series of other activities, that the so-called Maintenance Poles are accountable of carrying out technical investigations on incidents and accidents related to the material of its own competence. For the case under exam, the publication assigns to the Small Arms Maintenance Pole (SAMP) the competence on small arms and light weapons. 
The publication defines Transport as an operational activity aimed at transferring personnel, vehicles and materials from a place of origin to a destination, using land, sea and air carriers, through the related infrastructure.

In the second section, the ILE-MM defines the Park Materials as the union of all weapons, artillery, technical means for shooting, rotated and tracked vehicles, machines for earthworks, and aircraft supplied to the Army. It identifies five Park Areas and respective sub-areas, including homogeneous sets of several species of materials, means or weapon systems, having similar technical and employment characteristics, unitarily considered for the purpose of facilitating the material management. For the purposes of this work, the "E Area" concerning armament materials is the one that interests us the most.

\subsubsection{ILE-NL-2100-0006-12-00B01}

The publication ILE-LM is one of the dossiers of the ILE described in Section 2.2.1 and concerns the materials relating to the "E Area" Park, for which it defines the management activities and the related logistic device. For each material, a specific technical life is indicated in order to allow a correct management of the potentials as well as the planning of maintenance operations. At the end of this life, the material to be sent to the Maintenance Pole is submitted to general revision and, whereas possible, to carry out the resetting of the technical life. The publication then reports a series of tables, for each type of weapon, which detail the preventive interventions, the competent Logistics Area (FLA or FLS), the periodicity of these interventions, the necessary time.

\subsubsection{TER-50-1000-0007-12-00B000}

This document details the technical inspection procedures aimed at ascertaining, at all levels, the usability of the arms allowing both safety for the human user and reliability for operational purposes. The verifying instruments are a set of artefacts that allow for the checking of significant dimensions for the most important parts of the weapon from the point of view of safety and operation in a simple and rapid manner, as designed by the weapon manufacturer.

\subsection{Focus of the Research: Reporting}

Following the previous mentioned regulations, the inconveniences constituting technical problems must be reported according to the form in the Annex O of the publication ILE-LM, which defines the roles and the action for each supply chain agent. The reporting form has to be sent to three agents: (i) the Land Armaments Directorate (LAD), which is responsible for technical-administrative issues related to armament, (ii) the Command of Transport and Materials of the Army Logistic Command, (iii) the Logistic Department of the Army General Staff, and (iv) to the Commands on which the report producer unit depends. The report is structured in five sections, which are described following a supply chain information management perspective.

The first section deals with the description of the material and the parts affected by the problem, as well as the applicable technical documentation and the data related to the various types of maintenance interventions carried out. From an information management perspective, it is relevant to prioritize some of fields included in the technical report.

For the material, relevant fields are the Unique Codification Number (NUC), the name and type of the weapon, the Manufacturer, the number plate/frame/serial number (or other identifying element), the date of introduction into service;

For the system: the name, the serial number, the sub-group or the figure number, while for the parts involved in the incident: the name, the catalogue number and the NUC. Additional fields are the relevant Publication or Technical Ordinance; dates and number of hours of operation, and number of shots fired before the last specialized maintenance, the last reliability check, the last technical check, as well as the most recent general revision.

The second section illustrates the purpose of the reporting, highlighting whether it concerns the safety of personnel; regards the functionality of the material; is for information purposes only. 
The third section deals with the description of the problem, including shots/hours of usage when incident happened; narrative of the incident; possible causes (if any); taken measures (if any); number of detected cases (if any); previous reports (if any); proposals (if any); any other note.

The fourth section expresses the reporting priority (from high to low): emergency; urgent; normal.

The fifth section (not mandatory) contains a field to report any other data considered to be of interest, and some photos of relevant parts are usually attached, as shown in (e.g.,) Figures 2 and 3.

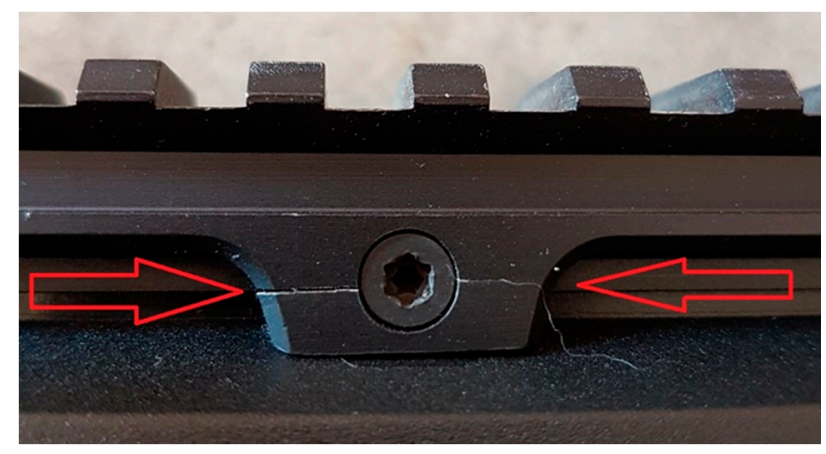

Figure 2. Example of technical inconvenience: mechanical damage in the rail of automatic rifle.
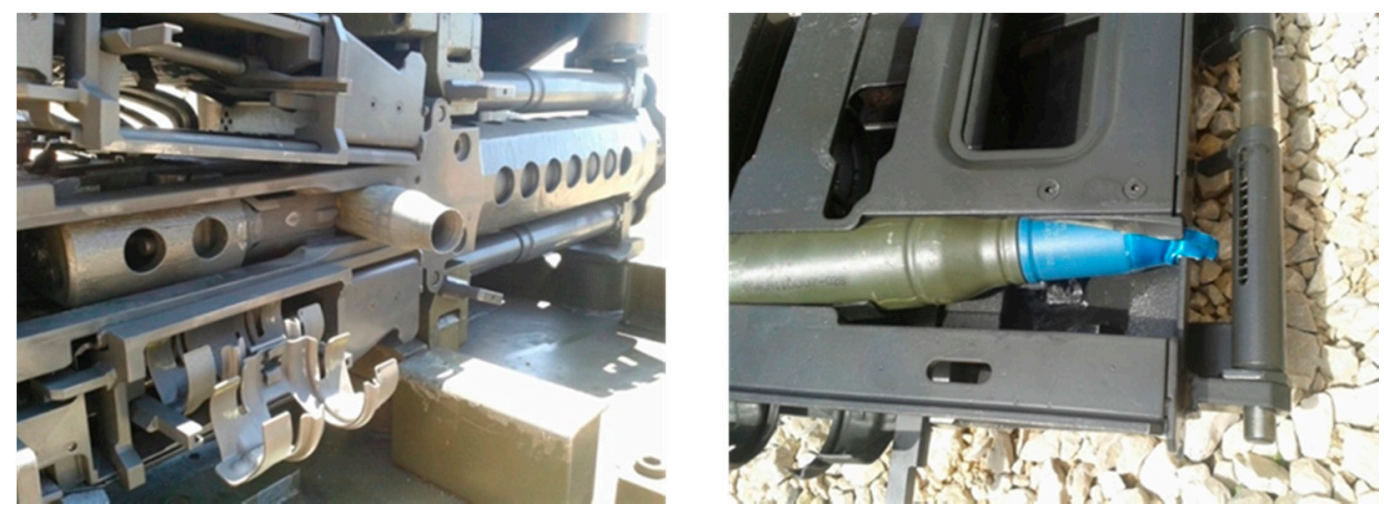

Figure 3. Example of a technical inconvenience for cal. $25 \mathrm{~mm}$ machine gun: jammed munitions during the feeding cycle).

Nevertheless, the information management from these reporting activities does not prescribe any systemic data structure and data collection, lacking treats for a completely effective LFI system. Starting from such approach, Section 3 presents a centralized SCM approach in order to maximize the potential of the LFI system, whose results are presented in Section 4.

\section{The Proposed Approach}

The centralized information management system was developed for the SCM of small arms, and it is here described in terms of data collection (Section 3.1), and database structure (Section 3.2). Then analysis on the dataset are presented in the context of a specific weapon (Section 4), which is supplied to three different Armed Forces. Data and analyses refer to a 6-year interval span, which were manipulated in order not to break the intellectual property of the agents involved, without disclosing any sensitive information.

\subsection{Centralized Supply Chain Information Management: Data Collection}

The first phase of the work was the data collection from the submitted forms, creating an IT data repository. The report proposed by the ILE-LM should be rigidly structured with well-defined fields to facilitate data entry and allow a simplified information management. Nevertheless, the acquisition, 
collection, and management of the reports for the weapon of the case study was a long and laborious activity, which lasted for about six weeks. The reason for this process lies in the way in which the messages and the related forms have been transmitted over years, i.e., the oldest reports were in paper format and it was necessary to consult the historical archive and manually enter the data in the various fields of the database. For this reason, a centralized digital repository became necessary and motivated the development of a Business Intelligence (BI) solution to ease dynamic data extraction and data analyses. We want to further stress this point, since it suggests an added value for the paper at hand, clarifying how the contributions.

\subsection{Database Structure}

Once the reports were gathered, it was necessary to structure the received data in a relational database. Such a database was built to allow an immediate data consultation and support holistic analyses in a multi-variate perspective. This database, whose architecture is represented in Figure 4, is made up of five tables: Reports, Weapons, Units, Contracts, Geographical coordinates. Note that the presented structure is intended as a general data architecture to be used for BI. Figure 4 aims to put emphasis on data organizations in tables, and relationships among tables for the subsequent analyses.

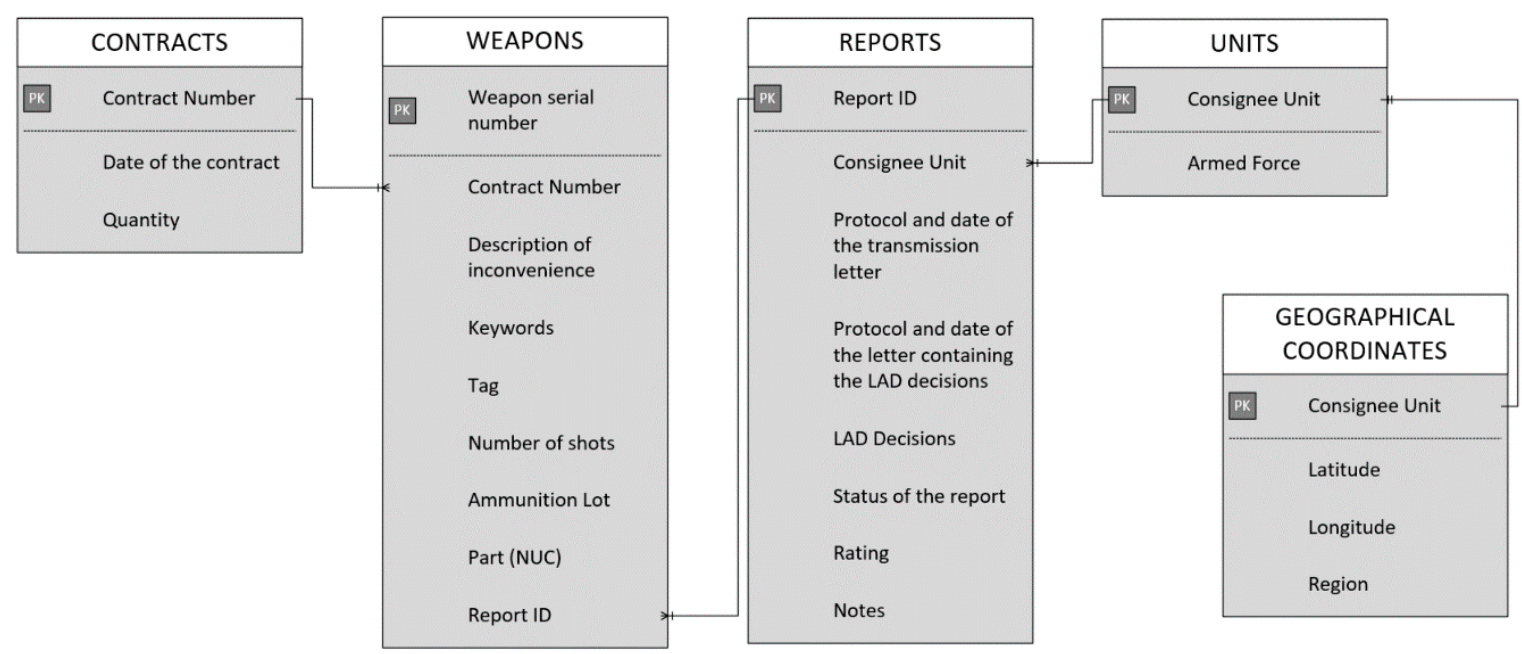

Figure 4. Database architecture using the Crow's foot notation (PK = Primary Key).

The "Reports" table contains all the reports received over a period of six years, theoretically, by units belonging to three different Armed Forces. The key of the table is the couple protocol and date of the reporting message. Other fields of the table are: report identification number (primary key); consignee Unit (or originator of the report); protocol and date of the LAD technical inquiry format letter; protocol and date of the transmission letter of the SAMP technical investigation; protocol and date of the letter containing the LAD determinations; LAD decisions; status of the report; Rating (a score calculated following the rules in Section 4.2); and a free text for notes. The "LAD decisions" field contains the decisions taken by the LAD. In case no investigation is considered necessary, the motivations are reported or, once the investigative process was completed, the corrective actions identified or undertaken are described; The "status of the report" field: it can be "in process" if LAD has still to decide, "in progress", if the technical investigation is being carried out at the SAMP, "closed" when the SAMP closed the investigation and LAD has provided its determinations or the technical investigation is not necessary since the inconvenience is already known or because it is due to the normal use of the weapon;

A "Weapons" table, which shows the data recorded on the forms for each weapon, was also implemented. This table is linked to the previous one using the same key, i.e., the couple protocol-data. Besides the key, its fields are: weapon serial number (primary key); number of the contract; description 
of the inconvenience; keywords; tag (label used to categorize the incident/accident); number of fired shots; ammunition lot; and part. Note that the ammunition lot is a fundamental information, particularly relevant in cases where an explosion of the weapon has occurred. Unfortunately, the standard reporting form does not provide a dedicated field and, thus this information is not always reported (requiring a successive dedicated information request).

The "Units" table concerns the originators of the reports and includes the following fields: Consignee Unit (primary key); AF (indicating the unit's Armed Force).

The "Geographical Coordinates" table details the latitude and longitude of the unit, to support a graphical representation of the reports in relation to Units. This table comes as a separate table from Unit since it includes data already in place before the development of the LFI system.

The "Contracts" table contains details on the contracts for the supply chain of the analyzed weapon. Relevant fields are: contract number; date of the contract (the date of the contract's approval); quantity (the number of weapons procured with the contract).

\subsection{Business Intelligence Framework}

Once the data were organized according to the structure described in Section 3.2, statistical analyses were performed to support a holistic and effective supply chain information management. These analyses rely on Business Intelligence (BI), intended as a set of techniques, technologies, systems, practices, methodologies, and applications that transform raw data into meaningful and useful information. BI was adopted in several domains, with recent research developments in aviation safety LFI at European level [41,42]. Similarly, BI becomes relevant for modern supply chains, since it supports a deeper understanding of organization' business values [43]. To this extent, the proposed LFI system, implemented via a BI architecture supports a large number of analytical analyses and a dynamic investigation of the variables included in the dataset, filling a gap in available literature on reverse supply chain in military environments.

\section{Results}

This section describes the main results obtained from the application of the BI approach based on the LFI perspective to a case study.

\subsection{Preliminary Observations on Reporting Activity}

As a preliminary observation, the reports of technical incidents/accidents related to the case study originated by Units belonging to two different Armed Forces. Since the weapon at hand is supplied - albeit in minor quantities - even to a third Armed Force, it is questionable that no reports were sent by the latter one.

Another consideration that can be made on reporting activity with reference to the case study, is related to the number of Units that have reported technical problems. This number represents about a third of the Units that actually make use of the weapon at hand. The issue to be investigated should explore if the absence of reporting by approximately sixty percent of units is justified (e.g., particular precautions taken during training or maintenance procedures that allowed preventing incidents), or is due to under-reporting (i.e., in case of an incident, the weapon is sent for repair or replacement, if under warranty, directly to the manufacturer, without reporting the incident following relevant regulations).

\subsection{Systematic Analysis of the Reports}

Firstly, the analysis showed that just less than $10 \%$ of the reports were compiled correctly in each section. More specifically, 35\% did not include the shots number; and 90\% did not contain any indications on the lot number of ammunitions. It is important to note that this latter information was always reported in cases that presented an explosion of the weapon, or was acquired successively upon explicit request by the LAD. Additionally, $15 \%$ of the reports did not include either the name 
of the item affected by the incident, nor the NUC; the same serial number was reported in six cases, which indicates an error in the reporting form (same weapon uses multiple times).

Starting from these observations, a rating system was calculated for each form to support holistic information management and the goodness estimation of reports. The purpose of this type of analysis consists of identifying the most virtuous reporting Units and taking them as reference examples (best in class, see Figure 5) for the other Units, to push them to produce reporting forms with increasingly complete and accurate information. The rating includes three families (Number of shots reported, Ammunition lot reported, NUC reported) with maximum assigned scores respectively $(30,30,40)$.

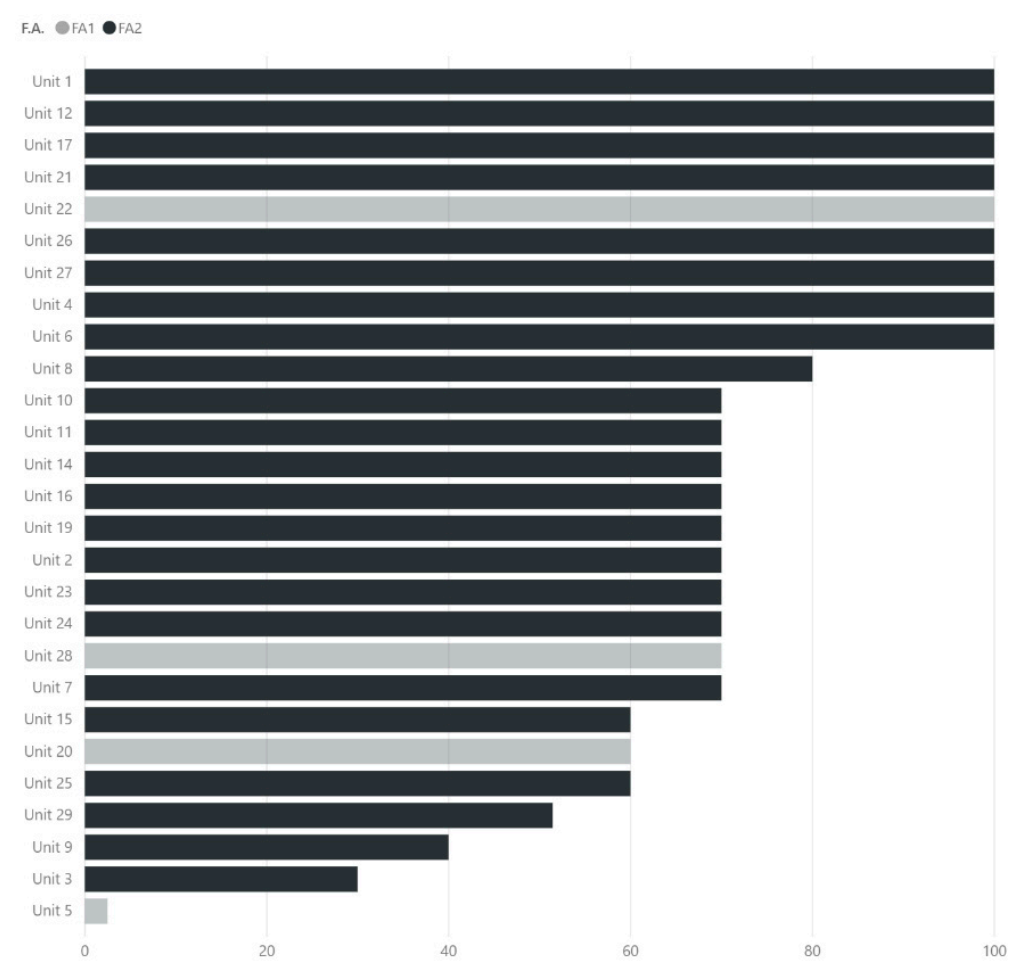

Figure 5. Scores distribution of reports' quality per unit.

\subsection{Relationships Between Contracts and Incidents}

Subsequently, the six procurement contracts were analyzed in relation to the number of reports to map the contractual supply performance. The relationships were discussed in relative terms, i.e., number of reports vs. number of weapons supplied through the contract. The contract presenting the highest report/quantity ratio is the first contract in chronological order (Contract 1 ) with a decreasing report/quantity ratio in accordance with the time span (see Figure 6). Such contract refers to a product which was undergoing consolidation, i.e., the first phase of its usage with multiple problems emerging. Over the years, the adoption of mitigating actions defined through the reporting process motivated changes to the configuration of the weapon to improve its technical maturity (Contract 8 and 9). 


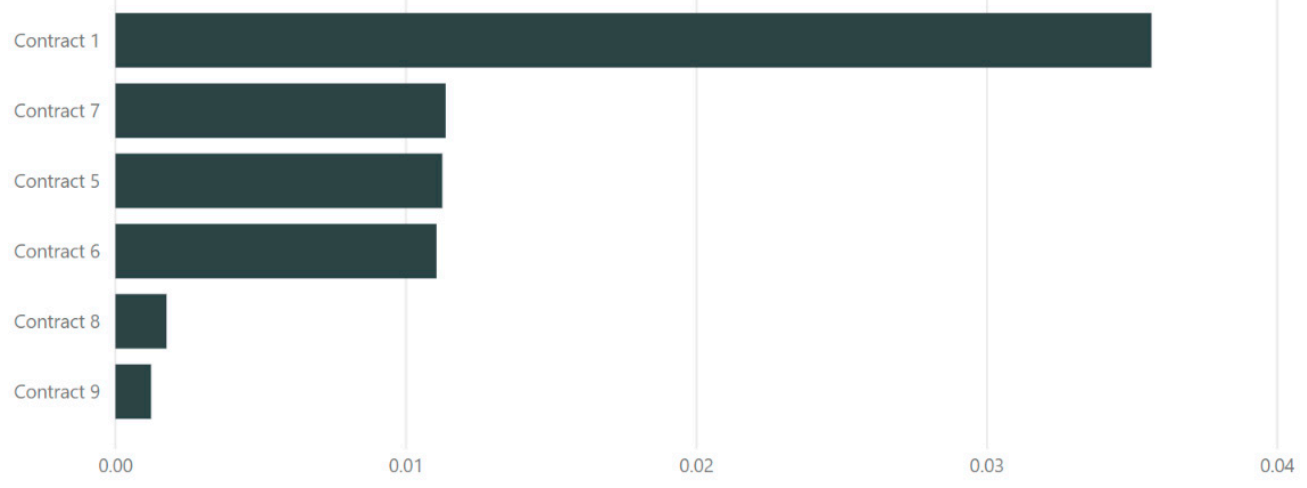

Figure 6. Reported cases over quantities per contract.

\subsection{Pareto Analysis}

A Pareto analysis is here used as a tool to prioritize some aspects of the phenomenon being investigated out of multiple causes, which compete to gain attention. In more operational terms, firstly, each category of incidents/accidents occurred was tagged: a "tag" was added to each report, containing a three-letter code to link the incidents belonging to the same type (see Table 1).

Table 1. Tag codification for main failure modes.

\begin{tabular}{|c|c|}
\hline TAG & DESCRIPTION \\
\hline GAS & $\begin{array}{l}\text { (Gas recovery system failure)-The label identifies cases where it was found or there is a } \\
\text { suspicion that the jams occurred on the weapons are due to the non-compliant diameter of } \\
\text { the hole in the gas outlet that regulates the recovery system to subtract gas }\end{array}$ \\
\hline HDL & (Handle failure) - Tag used to identify all cases that have a defect in the front handle \\
\hline INV & $\begin{array}{l}\text { (To Be Investigated)-Tag used for those cases to be investigated, whose reasons have not } \\
\text { yet been clearly identified but have in common the occurrence of an explosion of part of } \\
\text { the weapon }\end{array}$ \\
\hline MEC & $\begin{array}{l}\text { (Mechanical failure)-Tag used to identify mechanical failure of a component, not related } \\
\text { to an explosion phenomenon }\end{array}$ \\
\hline NCD & $\begin{array}{l}\text { (Non-Conformity to Drawing)-Tag used to define the cases in which a non-conformity } \\
\text { issue is found with respect to the constructive drawings on some details of the weapon }\end{array}$ \\
\hline NUL & $\begin{array}{l}\text { (Null) - Tag used to identify cases of minor interest that do not constitute a serious } \\
\text { inconvenience }\end{array}$ \\
\hline SLD & $\begin{array}{l}\text { (Rail slide failure)-Tag used to identify all cases that have a defect in the upper guide of } \\
\text { the slide }\end{array}$ \\
\hline SPR & $\begin{array}{l}\text { (Spring failure)-Tag used to identify all cases where there is a defect in the springs of the } \\
\text { ejector-ejector assembly }\end{array}$ \\
\hline THM & $\begin{array}{l}\text { (Thermo-fusion failure)-Tag used for cases in which a principle of thermo-fusion of the } \\
\text { polymer constituting the carcass has occurred. }\end{array}$ \\
\hline USC & (Partial Unscrewing failure)—Tag used to identify cases of partial unscrewing \\
\hline
\end{tabular}

Using the Pareto diagram in Figure 7, it is possible to note that three categories (i.e., SPR, SLD, GAS) out of ten cover more than $75 \%$ of the identified technical inconveniences. This type of analysis allows thus isolating the technical inconveniences where priority interventions is required. It also allows for an immediate ex-post verification of the effectiveness of the implemented interventions: by comparing two representations of the same phenomenon, before and after the intervention, there is a clear overview of the progress made and a measure of overall improvement. 




Figure 7. Pareto's diagram applied to most relevant technical components.

In the case study at hand, if a Pareto diagram is re-calculated (see Figure 8) considering only the reports whose status were "in progress "or "in process", it can immediately be observed that the cases relating to the three above-mentioned categories (i.e., SPR, SLD, GAS), are no longer included in the new diagram. This observation confirms that the majority of reports do not represent a current issue and they were solved in more recent contracts.

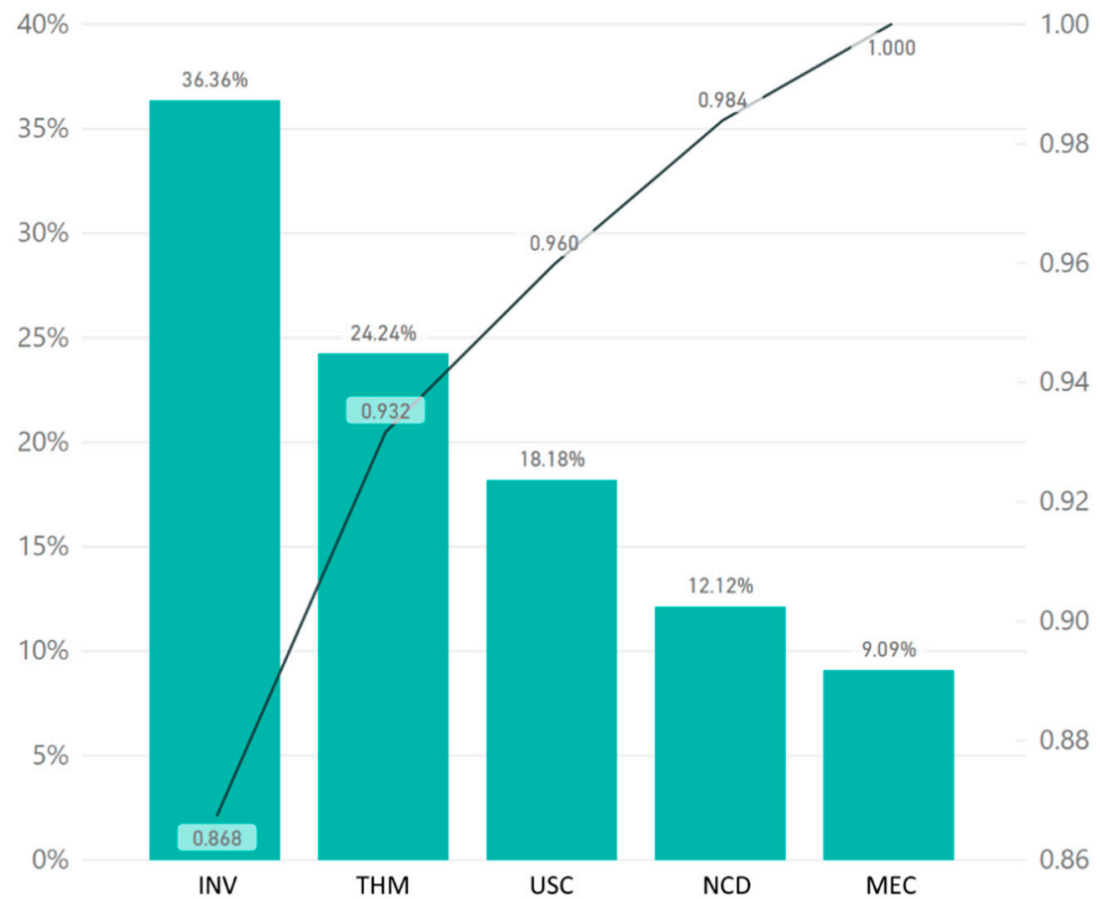

Figure 8. Pareto's diagram applied to most relevant technical components (only "in progress" or "in process" status). 


\subsection{Dynamic LFI Dashboards}

With the purpose of creating a dynamic business intelligence tool for the LFI system, further information was integrated into a dynamic dashboard (see Figures 9 and 10). More specifically, Figure 9 shows some summary data regarding the inconveniences divided into categories: (top left) a histogram depicting the distribution of the incidents by category, (bottom left) a sketch of the same incident categories per involved Unit, (right) a geographical map with a bubble pie chart where the size of the bubble presents the total number of reports, broken down per report tag. The dynamicity is developed through the implementation of the database structure into Microsoft PowerBI, which can be interfaced with the majority of data sources, ranging from MS Excel and MS Access to SQL, IBM database, and more.

Note that the dashboard is not static in the sense that the user can click on any point of any visual to select only one or more elements and automatically query the database to get the respective filtered/combined information.
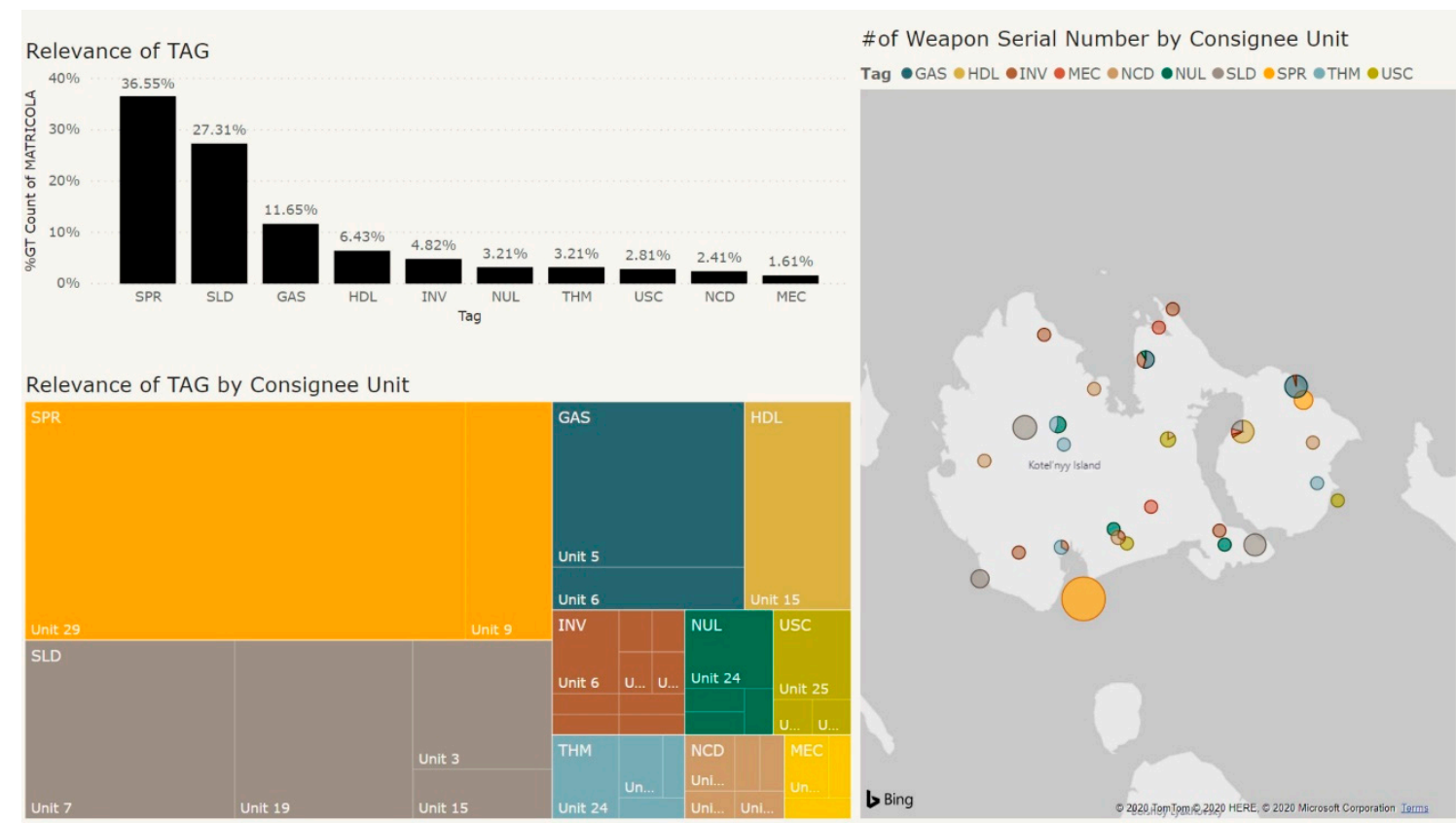

Figure 9. Main dashboard page of the integrated LFI system (quantitative and geographical data were manipulated in order not to break the intellectual property of the units involved).

As a second example, another dashboard page is proposed to show the potential of the framework, and its feasibility to accommodate different type of analysis and different visualizations.

Figure 10 presents a panel where the top right pie chart details the count of Weapon Serial Number by Contract Number, and the respective average rating of technical report. An average target of 90 out of 100 points per report is reported in the gauge (bottom left), while the overall average value is 73 . Furthermore, the bottom right graph presents a decomposition tree addressing the count of Weapon Serial Number that were reported in one or more report, and decomposing this number by the different Armed Forces, Contract Numbers, Consignee Unit, and Tag. In the presented case, the analysis stops at Unit 15, clarifying how the reports issued by this unit, referred to Contract 5 involved in descending order Handling failure (HDL), Rail slide failures (SLD), Mechanical failures (MEC) and a small portions of aspects to be further investigated (INV). This dynamic dashboard is intended as a support tool for the decision-maker for managing data for the supply chain at hand. 


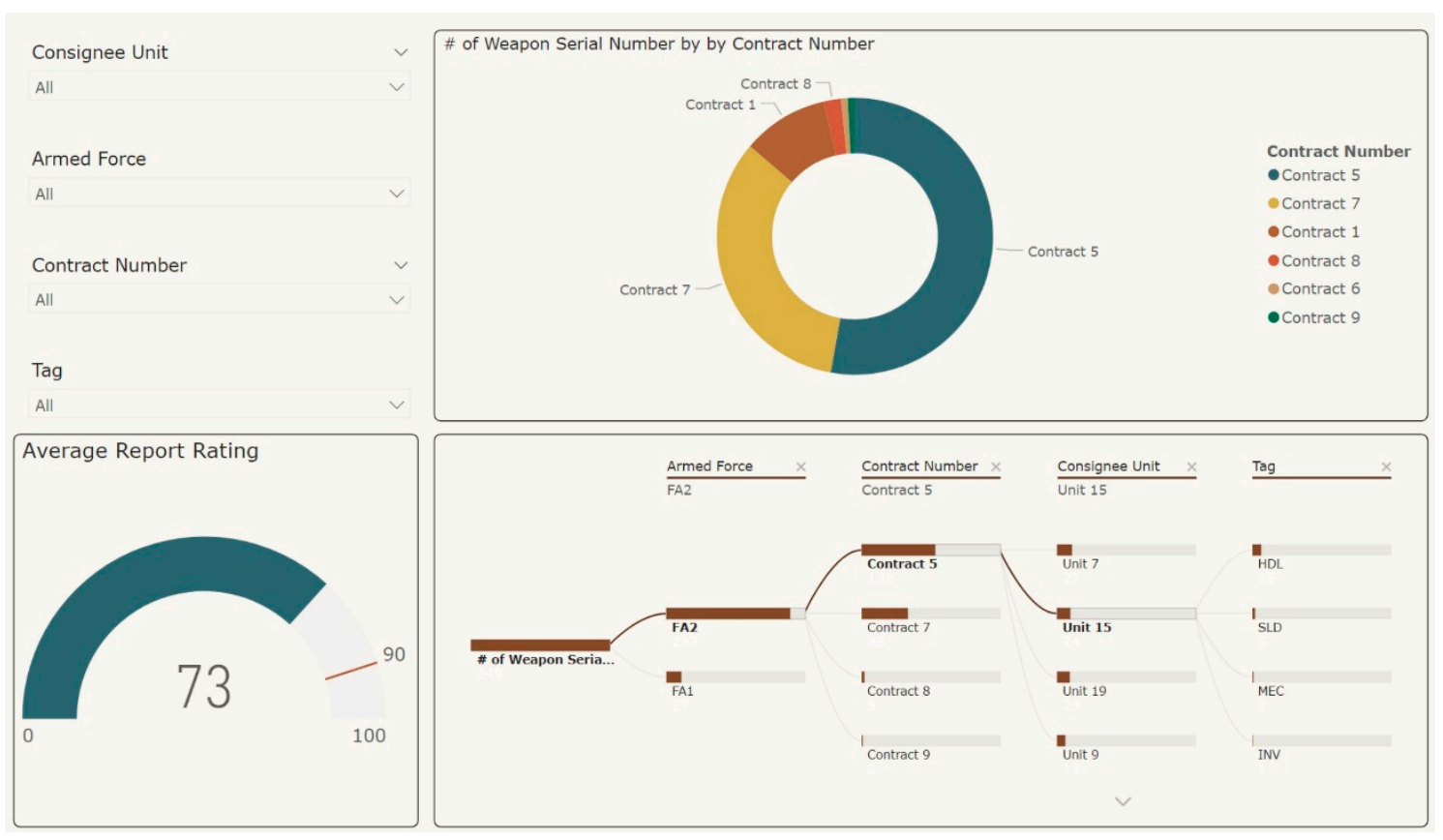

Figure 10. Detailed page on reporting of the integrated LFI system (quantitative and geographical data were manipulated in order not to break the intellectual property of the units involved).

\section{Discussion}

Following the analysis presented in Section 4, it is possible to outline improvements aimed at ensuring a sustainable SCM via the proposed LFI system.

\subsection{Prioritization of Reporting}

In order to promote and encourage reporting by all the Armed Forces and Armed Corps on the technical incidents related to the weaponry material, it is desirable to issue an inter-forces technical publication that regulates the process. For example, in Italy still, for the aeronautical sector, the technical publication AER (EP).00-01-6a Ed. 2010 "Instructions for the compilation, forwarding and management of inconvenience reports related to aeronautic material", issued by the Aeronautic Armaments Directorate (AAD) is used as a normative example in this direction. The purpose of the AAD publication is to regulate, through the issue of Inconvenience Reports, the activities related to the flow of information and materials so that all the necessary actions are taken and implemented to determine the causes of the occurrence of incidents/accidents related to the aeronautic material. This standard is applicable to all aircraft configuration articles registered in the Register of Military Aircraft of the AAD in relation to the production and use phases.

A similar solution could be adopted in the reporting process concerning the armament material, providing a centralized and unitary management of the incidents related to weapons. In addition, another cue for reflection about the aforementioned AAD publication, is the distinction of the Inconvenient Report in two different categories, which activate two different prioritizations of procedures (based on information, timescale). For the most critical reports (reporting defect or inconveniences of immediate danger that impacts airworthiness and safety for people or things) the AAD publication envisages the immediate involvement of the Design Authority. This shall react immediately within three working days, formulating precautionary and/or definitive measures to ensure that unacceptable operations are protracted over time. Such an approach should undoubtedly be followed when accidents related to weaponry material have a significant impact on safety. 


\subsection{Improving Digitalization}

The data collection phase for the application of the method was an extremely time-consuming phase, since several documents were still paper-based, or there were scans of paper documents hardly processable by automated IT systems (i.e., OCR systems). For this purpose, it could be beneficial to create an IT data entry form, or more suitably, a dedicated web portal on the intranet of relevant military authority. This portal should have a section where one can upload all the information related to the technical problem, by filling out a digital reporting form. It could be possible to have automatic quality check for the report completeness and obtain real-time statistics for the supply chain status. In this phase, a further recommendation would be linked to the formalization of the incident description field, that, being narrative, would benefit from the introduction of a taxonomy.

The taxonomy should allow for the identification of (at least) the affected part of the weapon, the failure mode, its effect, and the use condition. On this path, the taxonomy could be developed in a data-driven approach to reduce the efforts for its development. Such a data-driven approach would lead to the extraction of relevant information from the reports at hand by machine learning techniques for data extraction and text mining, (e.g., Naïve Bayes Classifiers) [44,45]. Once the taxonomy is developed, the "Description of the inconvenience" data field could be potentially structured providing auto-completing text, or even the allowing of the choice only among a determined set of options, based on the data gathered so far that feeds the taxonomy. Obviously, for each field, there should also be an option for an "other" choice that provides the possibility to add information that is not included in the previous list. Such extra data should be revised on a regular basis and, if relevant and significant, added among the permanent selection options, integrating the taxonomy itself. Consequently, the taxonomy could become incrementally wider and more representative, with the advantage of having a uniform database, to allow further text-based analytics.

\subsection{A 4.0 LFI System}

An additional technology in favor of digitalizing the reporting process could be related to the introduction of an intelligent weapon management system, in line with recent Industry 4.0 notions, propelled by the upsurge and progressive maturity of new information technologies [46]. An intelligent armory management system would facilitate operations through the entire supply chain. Exemplary areas affected by a smart weapon management system would be: (i) the control of access in the armory based on different privileges and the management of the authorization to take arms, providing an undoubted improvement of the process in terms of security; (ii) the logistic and maintenance management of weapons in terms of the potential expressed by the weapon taken from the armory and the management of any maintenance needs following anomalies or malfunctions, as well as the management of preventive maintenance; (iii) the management of accidents/incidents related to the armament material in real-time.

Regarding the latter point, the smart system might be interfaced with the portal discussed in Section 5.2, to feed the underlying database favoring and simplifying the reporting process. A series of data could in fact be acquired directly from the weapon, reducing the efforts for the data entry. This would be possible if each weapon had an electronic device, integrated into the weapon, that allows the recording of the number of shots fired, like the odometer used in cars, and RFID (Radio-frequency identification) tags on which to store information such as the model, the serial number, the contract, the number of shots.

It is easy to picture the improvement that would follow the introduction of such an intelligent armory management system in terms of the weapons maintenance process, since it would guarantee the acquisition of a large number of returns from the operational field, which is an essential starting point to proceed in the studies on reliability and maintainability of the various types of weapons. 


\subsection{A Healthy Reporting Environment}

Incident reporting starts any LFI system. However, other latent factors ensure the LFI initialization even earlier. It is important to observe how an increase in the safety level should not be connected to a decrease in the number of reported incidents, but rather to their lower severity: in other words, few reports do not imply a higher safety level. This situation could be rather a consequence of under-reporting, due to an unhealthy working environment. As for the wide literature in the safety area, the fear of being punished or accused of negligence in managing the weapon could discourage a reporting action. The participation of personnel involved in the investigation activity could be favored in a periodic toolbox meeting at the units responsible for reporting, in order to show the benefits of reporting by means of some significant incidents and recommendations.

On this path, expanding the functionalities of the portal discussed in Section 5.2, the same portal could be further used to gather information on the most significant incidents/accidents, illustrating the investigative activity carried out and the corrective actions (e.g., revision of procedures, emissions of technical notes, execution of retrofit interventions). Such approach may further advance the spreading of the recommended actions, contributing to the overall LFI system via a healthy reporting culture. This latter point puts emphasis on understanding work as actually carried out at organizational level, and on empowering sharp-end operator knowledge [47], ensuring sustainable reverse logistics, and in general, systemic supply chain resilience [48].

\section{Conclusions}

The literature analysis about LFI emphasizes the importance and the need to implement an effective Incident Learning System in order to achieve higher safety levels within an organization. Through the analysis of the supply chain information management process on military technical inconvenience for armaments, this paper presented an LFI framework valid for military supply chains.

The analysis shows empirical evidence on how technical incident data acquire values that go beyond the technical investigation: a well-structured database can be the basis for effective decision-making, which can be supported by synthetic user-friendly and easily accessible dashboards. Using a Business Intelligence perspective, the developed LFI system shows the benefits of a centralized information management system and supports the identification of future developments beyond the company being analyzed, i.e., the development of an inter-forces reporting regulation, the enhancement of digitalization in the entire process, the adoption of smart systems, and the fostering of a healthy reporting environment. It is important to observe how this paper provides just a subset of the possible indexes to be used for monitoring supply chain sustainability in light of the LFI framework. Furthermore, additional quantitative analyses can be developed to extend the analytical dimension of the proposed framework, e.g., clustering algorithms, trend analyses, multi-dimensional correlations.

Regarding the intelligent data management system, one aspect not considered in this research is related to privacy issues and threats related to cyber-security, which opens the path to future research in the same context [49].

More generally, the proposed LFI system described in this paper could be further enhanced, analyzing the important connections with the world outside of the organization, i.e., exploring how local learning is shared outside the supply chain agents, i.e., providing an external cycle for shared learnings. This cycle could be closed by the benchmarking process that analyses best practices and adapts them to improve the military system in a sustainable perspective.

Note also that the approach refers to the Italian regulatory framework for military reporting, but since it is grounded in the general NATO learning doctrine, it remains flexible enough to accommodate the needs of other settings, as long as they rely on the same NATO foundations for systemic learning. Lastly, even though the proposed case study focuses on small arms, it is worth mentioning how the same logic remains usable for technical inconveniences related to other types of weapons and it could be thus reproduced for the analysis of early phases of the item lifecycle. 


\section{Acronyms}

$\begin{array}{ll}\text { AAD } & \text { Aeronautic Armaments Directorate } \\ \text { AF } & \text { Armed Force } \\ \text { GAS } & \text { Gas recovery system failure } \\ \text { HDL } & \text { Handle failure } \\ \text { ILE-LM } & \text { ILE Material Management (ILE-NL-2100-0006-12-00B01) } \\ \text { ILE-MM } & \text { ILE Logistic Management (ILE-NL-1110-0001-12-00B01) } \\ \text { INV } & \text { To be Investigated } \\ \text { LAD } & \text { Land Armaments Directorate } \\ \text { LFI } & \text { Learning from Incident } \\ \text { MEC } & \text { Mechanical failure } \\ \text { NCD } & \text { Non-Conformity to Drawing } \\ \text { NUC } & \text { Unique Codification Number } \\ \text { NUL } & \text { Null } \\ \text { SAMP } & \text { Small Arms Maintenance Pole } \\ \text { SLD } & \text { Rail slide failure } \\ \text { SPR } & \text { Spring failure } \\ \text { THM } & \text { Thermo-fusion failure } \\ \text { USC } & \text { Partial Unscrewing failure }\end{array}$

Author Contributions: Conceptualization, G.D.G. and R.P.; methodology, G.D.G., R.C. and R.P.; software, R.C. and R.P.; validation, R.C. and R.P.; formal analysis, R.C. and G.D.G.; investigation, R.C.; resources, R.C. and R.P.; data curation, R.C.; writing-original draft preparation, R.C. and R.P.; writing-review and editing, R.P. and G.D.G.; visualization, R.P. and G.D.G.; supervision, R.P. and G.D.G.; project administration, G.D.G.; funding acquisition, G.D.G. All authors have read and agreed to the published version of the manuscript.

Funding: This research received no external funding.

Conflicts of Interest: The authors declare no conflict of interest.

\section{References}

1. Kumar, K. Technology for supporting supply chain management. Commun. ACM 2001, 44, 58-61. [CrossRef]

2. Ballou, R.H. The evolution and future of logistics and supply chain management. Produção 2006, 16, 375-386. [CrossRef]

3. Iakovou, E. A new framework for supply chain management: Review concepts and examples. In Proceedings of the Third Aegean International Conference on Design and Analysis of Manufacturing Systems, TInos, Greece, 19-22 May 2001; pp. 27-36.

4. Erkan, B.; Mehmet, D.; Koh, S.C.L.; Tatoglu, E.; Zaim, H. A causal analysis of the impact of information systems and supply chain management practices on operational performance: Evidence from manufacturing SMEs in Turkey. Int. J. Prod. Econ. 2009, 122, 133-149.

5. Habib, M. Supply Chain Management (SCM): Its future implications. Open J. Soc. Sci. 2014, 2, $238-246$. [CrossRef]

6. Cigolini, R.; Cozzi, M.; Perona, M. A new framework for supply chain management: Conceptual model and empirical test. Int. J. Oper. Prod. Manag. 2004, 24, 7-41. [CrossRef]

7. Matinrad, N.; Roghanian, E.; Rasi, Z. Supply chain network optimization: A review of classification, models, solution techniques and future research. Uncertain Supply Chain Manag. 2013, 1, 1-24. [CrossRef]

8. Imran, M.; Hamid, S.; Aziz, A.; Hameed, W. The contributing factors towards e-logistic customer satisfaction: A mediating role of information technology. Uncertain Supply Chain Manag. 2019, 7, 63-72. [CrossRef]

9. Department of Defense. Fiscal Year 2017 Annual Industrial Capabilities; Department of Defense: Washington, DC, USA, 2018.

10. Gunasekaran, A.; Ngai, E.W.T. Information systems in supply chain integration and management. Eur. J. Oper. Res. 2004, 159, 269-295. [CrossRef]

11. Kim, S.; Cohen, M.A.; Netessine, S. Performance contracting in after-sales service supply chains performance contracting in after-sales service supply chains. Manag. Sci. 2007, 53, 1843-1858. [CrossRef]

12. Stock, J.R. Reverse Logistics: White Paper; Council of Logistics Management: Oak Brook, IL, USA, 1992. 
13. Khalili-Damghani, K.; Naderi, H. A mathematical location-routing model of repair centres and ammunition depots in order to support soldiers in civil wars. Int. J. Manag. Decis. Mak. 2014, 13, 422-450.

14. Qrunfleh, S.; Tarafdar, M. Supply chain information systems strategy: Impacts on supply chain performance and firm performance. Int. J. Prod. Econ. 2014, 147, 340-350. [CrossRef]

15. Hugos, M. Essentials of Supply Chain Management, 2nd ed.; Wiley: Hoboken, NJ, USA, 2006.

16. Fan, C.; Fan, P.; Chang, P. A system dynamics modeling approach for a military weapon maintenance supply system. Int. J. Prod. Econ. 2010, 128, 457-469. [CrossRef]

17. Liu, B.-P.; Zhang, K.; Huang, D. Constructing strategy of stable reverse military logistics system based on non-cooperative game. In Annual Conference Proceedings, Proceedings of the International Conference on Management Science and Engineering; Nomi, Japan, 17-20 August 2017, Tao, M.A., Zhen, S., Eds.; IEEE Computer Society: Washington, DC, USA, 2018; pp. 33-38.

18. Wang, K.; Jiang, D.L.; Liu, J.; Zhang, L. Waste military air material reverse logistics system multi-objective incentive and monitoring mechanism. Adv. Mater. Res. 2012, 518-523, 3631-3640. [CrossRef]

19. El Garrab, H.; Castanier, B.; Lemoine, D.; Lazrak, A.; Heidsieck, R. Towards hybrid machine learning models in decision support systems for predicting the spare parts reverse flow in a complex supply chain. In Information system, Logistics $\mathcal{E}$ Supply Chain-ILS, Proceedings of the ILS 2020 International Conference on Information Systems, Logistics and Supply Chain, Austin, TX, USA, 22-24 April 2020; The Center for Direct Scientific Communication (CCSD): Austin, TX, USA, 2020; pp. 188-195.

20. Mahmoudzadeh, M.; Mansour, S.; Karimi, B. To develop a third-party reverse logistics network for end-of-life vehicles in Iran. Resour. Conserv. Recycl. 2013, 78, 1-14. [CrossRef]

21. Glicksman, H.; Penn, M. Approximation algorithms for group prize-collecting and location-routing problems. Discret. Appl. Math. 2008, 156, 3238-3247. [CrossRef]

22. Carnes, T.; Shmoys, D.B. Primal-dual schema and Lagrangian relaxation for the k-location-routing problem. Lect. Notes Comput. Sci. (Incl. Subser. Lect. Notes Artif. Intell. Lect. Notes Bioinform.) 2011, 6845, 99-110.

23. Guerrero, W.J.; Prodhon, C.; Velasco, N.; Amaya, C.A. Hybrid heuristic for the inventory location-routing problem with deterministic demand. Int. J. Prod. Econ. 2013, 146, 359-370. [CrossRef]

24. Wilson, J.M.; Goodman, P.S.; Cronin, M.A. Group learning. Acad. Manag. Rev. 2007, 32, 1041-1059. [CrossRef]

25. Ramanujam, R.; Goodman, P.S. The challenge of collective learning from event analysis. Saf. Sci. 2011, 49, 83-89. [CrossRef]

26. Le Coze, J.C. What have we learned about learning from accidents? Post-disasters reflections. Saf. Sci. 2013, 51, 441-453. [CrossRef]

27. Lukic, D.; Littlejohn, A.; Margaryan, A. A framework for learning from incidents in the workplace. Saf. Sci. 2012, 50, 950-957. [CrossRef]

28. Silva, S.A.; Carvalho, H.; Oliveira, M.J.; Fialho, T.; Soares, C.G.; Jacinto, C. Organizational practices for learning with work accidents throughout their information cycle. Saf. Sci. 2017, 99, 102-114. [CrossRef]

29. Kaya, E.; Azaltun, M. Role of information systems in supply chain management and its application on five-star hotels in Istanbul. J. Hosp. Tour. 2012, 3, 138-146. [CrossRef]

30. NATO JALLC. The NATO Lessons Learned Handbook, 3rd ed.; NATO JALLC: Monsanto, Portugal, 2016; ISBN 978-92-845-0188-5.

31. Cooke, D.L.; Rohleder, T.R. Learning from incidents: From normal accidents to high reliability. Syst. Dyn. Rev. 2006, 22, 213-239. [CrossRef]

32. Cooke, D.L.; Dunscombe, P.B.; Lee, R.C. Using a survey of incident reporting and learning practices to improve organisational learning at a cancer care centre. Qual. Saf. Health Care 2007, 16, 342-348. [CrossRef]

33. Phimister, J.R.; Oktem, U.; Kleindorfer, P.R.; Kunreuther, H. Near-miss incident management in the chemical process industry. Risk Anal. 2003, 23, 445-459. [CrossRef]

34. Hayes, J.; Maslen, S. Knowing stories that matter: Learning for effective safety decision-making. J. Risk Res. 2014, 9877, 1-13. [CrossRef]

35. Dekker, S.; Pruchnicki, S. Drifting into failure: Theorising the dynamics of disaster incubation. Theor. Issues Ergon. Sci. 2014, 15, 534-544. [CrossRef]

36. Leveson, N. A new accident model for engineering safer systems. Saf. Sci. 2004, 42, 237-270. [CrossRef]

37. Hollnagel, E. FRAM: The Functional Resonance Analysis Method-Modelling Complex Socio-Technical Systems; Ashgate: Farnham, UK, 2012; ISBN 978-1-4094-4552-4. 
38. Pidgeon, N. The limits to safety? Culture, politics, learning and man-made disasters. J. Contingencies Cris. Manag. 1997, 5, 1-14. [CrossRef]

39. Sagan, S.D. The Limits of Safety-Organizations, Accidents, and Nuclear Weapons; Princeton University Press: Princeton, NJ, USA, 1995; ISBN 978-0691021010.

40. Rijpma, J.A. Complexity, tight-coupling and reliability: Connecting normal accidents theory and high reliability theory. J. Contingencies Cris. Manag. 1997, 5, 15-23. [CrossRef]

41. Patriarca, R.; Cioponea, R.; Di Gravio, G.; Licu, A. Managing safety data: The TOKAI experience for the air navigation service providers. Transp. Res. Procedia 2018, 35, 148-157. [CrossRef]

42. Patriarca, R.; Di Gravio, G.; Cioponea, R.; Licu, A. Safety intelligence: Incremental proactive risk management for holistic aviation safety performance. Saf. Sci. 2019, 118, 551-567. [CrossRef]

43. Chen, H.; Chiang, R.H.L.; Storey, V.C. Business intelligence and analytics: From big data to big impact. MIS Q. 2012, 36, 1165-1188. [CrossRef]

44. Liao, C.; Hiroi, K.; Kaji, K.; Sakurada, K.; Kawaguchi, N. Event locky: System of event-data extraction from webpages based onweb mining. J. Inf. Process. 2017, 25, 321-330.

45. Usai, A.; Pironti, M.; Mital, M.; Mejri, C.A. Knowledge discovery out of text data: A systematic review via text mining. J. Knowl. Manag. 2018, 22, 1471-1488. [CrossRef]

46. Diez-Olivan, A.; Del Ser, J.; Galar, D.; Sierra, B. Data fusion and machine learning for industrial prognosis: Trends and perspectives towards Industry 4.0. Inf. Fusion 2019, 50, 92-111. [CrossRef]

47. Patriarca, R.; Bergström, J.; Di Gravio, G.; Costantino, F. Resilience engineering: Current status of the research and future challenges. Saf. Sci. 2018, 102, 79-100. [CrossRef]

48. Lam, C.Y. Resilience of logistics network: Analysis and design. In Proceedings of the 2016 World Congress on Industrial Control Systems Security, WCICSS 2016, London, UK, 12-14 December 2016; Institute of Electrical and Electronics Engineers Inc.: Piscataway, NJ, US, 2017; pp. 62-66.

49. Chopra, A. Paradigm shift and challenges in IoT security. In Journal of Physics: Conference Series; Institute of Physics Publishing: Bristol, UK, 2020; Volume 1432.

(C) 2020 by the authors. Licensee MDPI, Basel, Switzerland. This article is an open access article distributed under the terms and conditions of the Creative Commons Attribution (CC BY) license (http://creativecommons.org/licenses/by/4.0/). 